\title{
LOW COST LASER RANGEFINDER EVALUATION
}

\author{
J. Krejsa*, S. Vechet ${ }^{* *}$
}

\begin{abstract}
Laser rangefinders are essential tools in indoor mobile robot navigation. Lately several low-cost models become available, enabling the use of this type of sensor in broader range of applications. This paper evaluates the properties of currently (2018) the cheapest sensor available, particularly RPLidar A1M8, in order to determine whether it is usable in mobile robot development. As the sensor is based on triangulation principle, the increase of errors with the measured distance was expected; the experiments proved these expectations. Furthermore, the increasing, but linear trend in absolute measurement error was observed. The influence of surface material and color was also evaluated. The measurements indicate that RPLidar A1M8 can serve as a useful alternative for more expensive types of laser rangefinders.
\end{abstract}

Keywords: laser range finder, mobile robot, localization

\section{Introduction}

Laser rangefinders are essential sensors for mobile robot mapping and localization, no matter the algorithm used, see, e.g., (Vechet 2007, 2014), as well as for obstacle avoidance. While localization can be solved by other means, for example using active beacons, as illustrated in Krejsa (2010), laser range finders are difficult to compete when the model of robot surrounding is being built, as proved by $\mathrm{Ye}$ (2002). The price and weight, together with power assumptions are the main limitations when low-cost applications are considered, however, recently a number of cheaper rangefinders became available, e.g. Hokuyo URG-04LX, whose properties were evaluated by Krejsa (2017).

Traditional laser rangefinders, such as those produced by Sick, use time-of-flight measuring principle. Hokuyo produced scanners use phase shift measuring principle. Further evaluated scanner RPLidar A1M8 uses triangulation ranging principle, enabling on one hand the substantial reduction in cost, but on the other hand the resolution of the scanner degrades with the distance measured. The aim of this paper is to evaluate the properties of the sensor to find out, whether such trade-off is acceptable for the low-cost small mobile robots developers and manufacturers.

\section{RPLidar A1M8 sensor}

RPLidar A1M8 (Figure 1) is produced by Slamtec company. Its measuring principle is shown in Figure 1 and its essential data sheet values are summarized in Table 1 . The scanner emits modulated infrared laser signal that is reflected by the object to be measured, and the returning signal is sampled and processed by the scanner to output the distance from the object. The scanner is mounted on a spinner that includes the angular encoding system, therefore the set of angle and distance pairs is returned during each revolution.

\footnotetext{
Assoc. Prof. Jiri Krejsa, PhD, Institute of Thermomechanics AS CR, v.v.i., Technicka 2, 616 69. Brno, Czech Republic, krejsa@fme.vutbr.cz

** Assoc. Prof. Stanislav Vechet, PhD., Institute of Thermomechanics AS CR, v.v.i., Technicka 2, 616 69. Brno, Czech Republic, vechet.s@fme.vutb.cz
} 

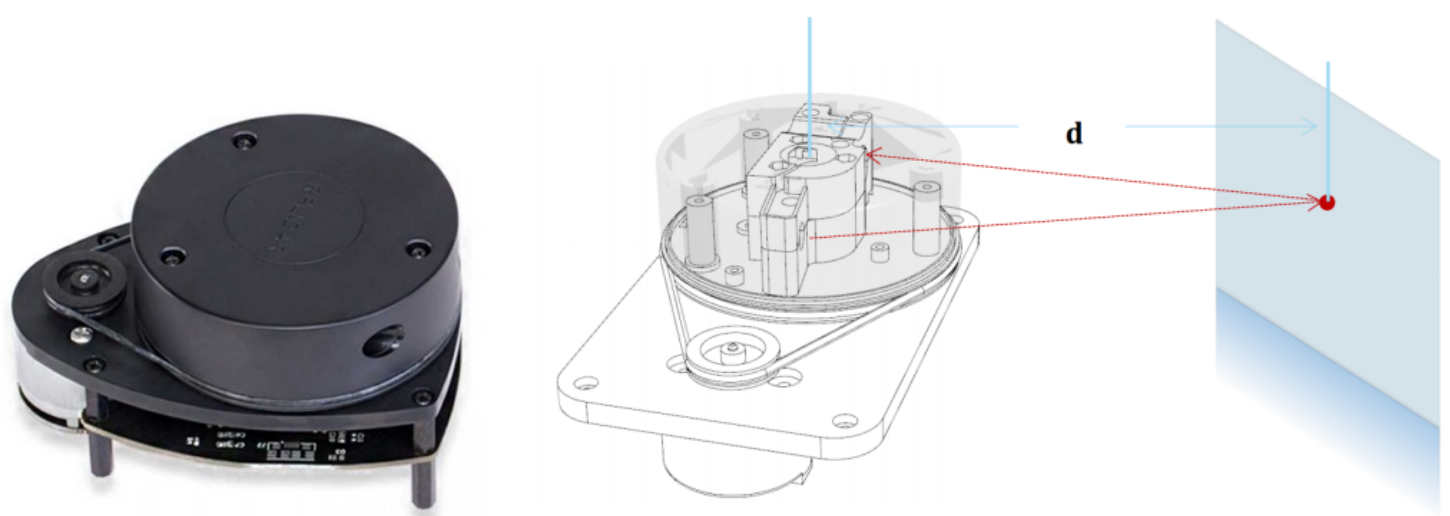

Fig. 1: RPLidar A1M8 sensor (left), its measuring principle (right) (figs. by Slamtec company (2018)).

Tab. 1: A1M8 RPLidar parameters

\begin{tabular}{cc}
\hline Parameter & Value [unit] \\
\hline Dimensions & $90 \times 70 \times 60[\mathrm{~mm}]$ \\
\hline Weight & $200[\mathrm{~g}]$ \\
\hline Measuring range & $200-6000[\mathrm{~mm}]$ \\
\hline Angular range & $360\left[{ }^{\circ}\right]$ \\
\hline Scan frequency & $10[\mathrm{~Hz}]$ \\
\hline Resolution & $<0.5[\mathrm{~mm}]$ \\
\hline Angular resolution & $<1\left[{ }^{\circ}\right]$ \\
\hline Temperature range & $0-45\left[{ }^{\circ} \mathrm{C}\right]$ \\
\hline Power consumption & $2[\mathrm{~W}]$ \\
\hline
\end{tabular}

\section{Measurement evaluation}

A number of measurements was performed in the following way. The sensor was set into defined position and aimed into the target. The output of the single scan contains the set of distances and the angle of particular beam. Single beam was selected, closest to the aimed direction and distance measured was corrected by triangulation. All the measurements were repeated and mean value and standard deviations were calculated.

\subsection{Distance measurement}

The most important property to evaluate is how the measurement error is influenced by the distance to be measured. Distance to the white wall, perpendicular to the scanner beam was measured repeatedly by two units. The measurement was repeated for the full set of distances to find out how stable the measurement is. The results are shown in Figure 2. Distance to be measured is on the $\mathrm{x}$-axis, measurement error, calculated as the difference between actual measurement and real distance is on the y-axis.

Figure 2 left shows the errors in repeated measurement by single scanner unit. The errors are positive in the whole range of measurement (scanner returns longer distances than the real ones). Both the error and standard deviation are increasing with the reference distance and we can see that the relation is linear. While the error of about $6 \%$ at the distance of $5 \mathrm{~m}$ is larger than expected, the linearity of error development enables simple compensation. The increase of the standard deviation was expected, it is connected with the measuring principle and there is not much we can do about it. 

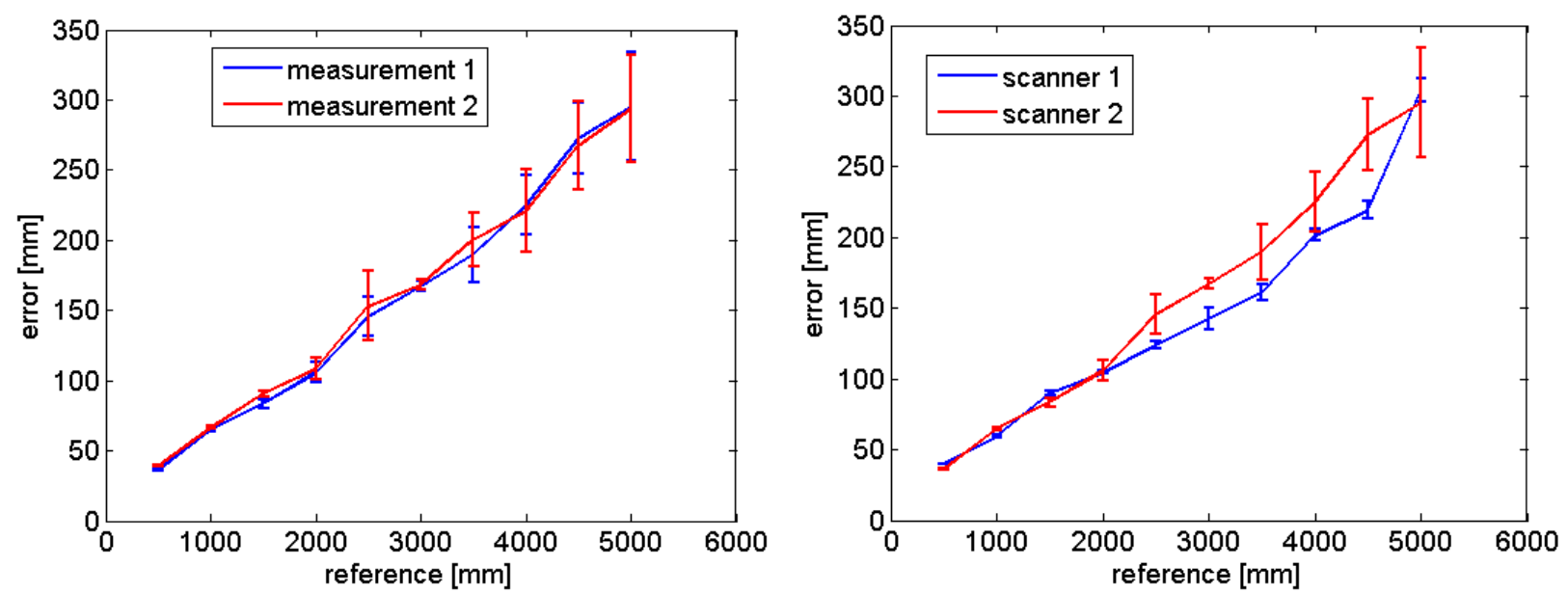

Fig. 2: Errors depending on the distance measured. Repeated measurements of single unit (left), measurements of two different scanner units (right)

Figure 2 right shows the same measurement, but performed by two different scanner units. While there are differences, we can see that the general linear trend in error development holds for both units and therefore can be compensated easily.

\subsection{Material color influence}

The influence of the color of surface was evaluated by repeated measurement at the reference distance of $1000 \mathrm{~mm}$. Results are shown in Figure 3. One can see that such influence is negligible, the difference between blue and green surface, which is the largest one, is about $3 \mathrm{~mm}$, way below the overall error in distance determination. Interesting, however, is the shape of curves, where for the green and blue there is clearly non-unimodal shape, while the rest resembles the Gaussian.

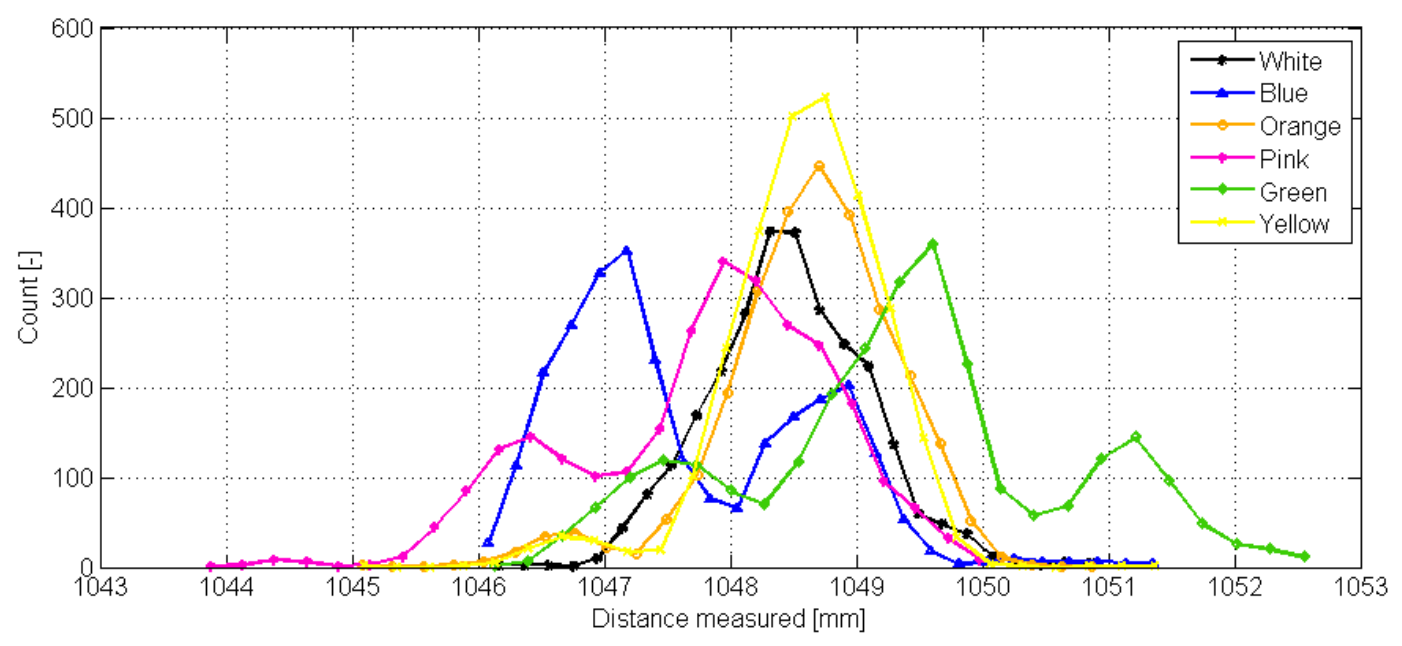

Fig. 3: Influence of material color

\subsection{Material influence}

The influence of the material was evaluated by repeated measurement at the reference distance of $1000 \mathrm{~mm}$. Results are shown in Figure 4. Materials common in indoor environment were selected for the experiment, including ABS plastic and high pressure laminate (HPL). White wall is taken as a reference. We can see that there is substantial influence of the material, and for some of them the shape of the curve is no longer any close to the Gaussian. For the glass we can observe two clear peaks, corresponding to the inner and outer surface of the glass (of the $7 \mathrm{~mm}$ thickness). For the HPL and ABS plastic the measurement is clearly scattered, for the reasons that are currently unknown. 


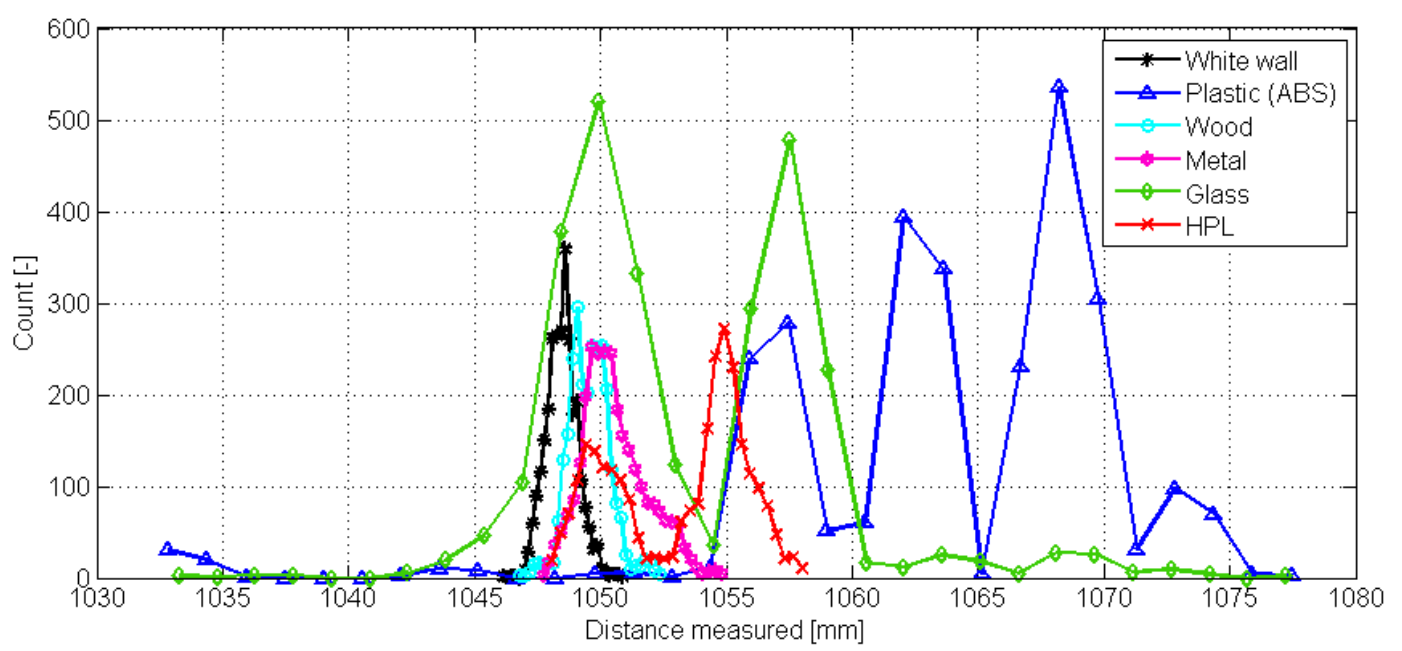

Fig. 4: Influence of material type

\section{Discussion and conclusions}

Experimental evaluation of RPLidar A1M8 shows the increasing measurement error over the distance measured. However, the trend in this error increase is approximately linear and therefore can be easily compensated. Two units were available for the authors, with the similar behavior, however that number is too small to conclude that the trend holds for this type of sensor in general.

RPLidar A1M8 is at the knowledge of the authors currently the cheapest 2D laser rangefinder available for on the shelf purchases, being roughly $1 / 4$ of the price of the Hokuyo URG-04LX scanner that was holding such position so far. The experiments indicate that while the triangulation principle necessarily degrades the measurement precision with increased distance, the overall performance of the sensor is sufficient for the most common tasks in indoor mobile robotics, such as obstacle avoidance, localization, SLAM and navigation as the whole. The only questionable feature is the presence of uncovered rotating parts, that might gain limitations in some applications.

\section{Acknowledgement}

The results were obtained with institutional support RVO 61388998 of the Institute of Thermomechanics AS CR v.v.i. The authors would like to thank Richard V. Krejsa, Filip Ferenc, Jiří Hanák and Radek Vobejda for their help with the experimental setup and measurements.

\section{References}

Krejsa, J., Vechet, S. (2010). Odometry-free mobile robot localization using bearing only beacons. Proceedings of 14th International Power Electronics and Motion Control Conference (Epe-Pemc 2010). DOI: 10.1109/Epepemc.2010.5606893

Krejsa, J., Vechet, S. (2017). The Evaluation of Hokuyo URG-04LX-UG01 Laser Range Finder Data, In: Engineering Mechanics 2017, Brno University of Technology, Brno, pp. 522-525.

RPLIDR A1. Slamtec [online]. [cit. 2018-01-02]. Available from: http://bucket.download.slamtec.com/e9e096e9d9f30205d665260abe2cfb0c2dd62efa/LD108_SLAMTEC_rplidar _datasheet_A1M8_v1.0_en.pdf

Vechet S., Chen KS., Krejsa J. (2014), Hybrid Navigation Method for Dynamic Indoor Environment Based on Mixed Potential Fields, Mechatronics 2013: Recent Technological and Scientific Advances, Springer, pp. 575582, DOI: 10.1007/978-3-319-02294-9_73

Vechet, S., Krejsa, J., Houska, P. (2007). The enhancement of PCSM method by motion history analysis. Recent Advances in Mechatronics, 107-110. Doi 10.1007/978-3-540-73956-2_22

Ye, C., Borenstein, J. (2002), Characterization of a 2D laser scanner for mobile robot obstacle negotiation, in Proc. of the IEEE Intrl. Conf. on Robotics and Automation, vol. 3, pp. 2512-2518 